\title{
Some Observations on Triplet Ground-States in the Context of 'Topological' (HLPM) Ring-Currents in Conjugated Systems
}

\author{
Timothy K. Dickens, Roger B. Mallion*
}

Peterhouse, Cambridge CB2 1RD, England, United Kingdom

* Corresponding author's e-mail address: mallionr29b@aol.com

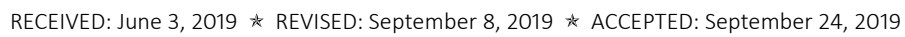

PROCEEDING OF THE $31^{\text {ST }}$ MATH/CHEM/COMP CONFERENCE, JUNE 10-14, 2019, DUBROVNIK, CROATIA

DEDICATED TO THE MEMORY OF DR EDWARD CAMERON KIRBY (1934-2019)

\begin{abstract}
When the quasi graph-theoretical Hückel-London-Pople-McWeeny (HLPM) approach is used to calculate 'topological' $\pi$-electron ring-currents and bond-currents in conjugated hydrocarbons, a problem is identified that occurs whenever application of the Aufbau process gives rise to a $\pi$-electronic ground-state configuration that is a triplet. This circumstance seems to occur only occasionally and, even when it does, the generally somewhat outré molecular graphs in question appear unlikely to represent extant or viable conjugated systems. The molecular graphs of four examples are used to illustrate this 'triplet ground-state problem', only one of which represents a hydrocarbon that has actually been synthesised. It is pointed out that the 'triplet ground-state problem' does constitute an intrinsic limitation of the HLPM approach. It is, though, a limitation that is also necessarily inherent in other equivalent (though ostensibly different) methods of calculating magnetic properties due to $\pi$-electron ring-currents - methods that are likewise founded on the Hückel molecular-orbital conventions. When a triplet ground-state arises, topological ring-currents and bond-currents cannot be calculated by the HLPM method and its equivalents. Infinite paramagnetism is formally to be predicted in such situations.
\end{abstract}

Keywords: Topological Ring-Currents in Conjugated Systems, $\pi$-Electron Triplet Ground-States, Aufbau Principle, Infinite Paramagnetism.

\section{INTRODUCTION}

$\mathbf{F}$ OR a period of some 25 years, one of us (RBM) was privileged to work with the late Edward Cameron Kirby (1934-2019), the honorand of this Session of MATH/CHEM/COMP 31 (Dubrovnik, 2019). A considerable proportion of our collaboration was devoted to the idea of spanning trees in graphs, ${ }^{[1-7]}$ especially of chemical graphs ${ }^{[8]}$ that represent conjugated hydrocarbons (extant or hypothetical). A prominent application of these ideas in Mathematical Chemistry has been in the context of what the present authors have named ${ }^{[9,10]}$ the Hückel[11,12]_ London $^{[13]}-P o p l e^{[14]}-M c W e e n{ }^{[15]}$ (HLPM) formalism for calculating what are frequently called 'topological' ringcurrents and bond currents ${ }^{[9,10]}$ - for reviews please see Refs. [16-19].

In the course of performing calculations on individual molecules by means of this HLPM approach a problem has, from time-to-time, been encountered whenever, on application of the Aufbau Principle, ${ }^{[11,20-24]}$ the $\pi$-electronic ground-state configuration of the conjugated hydrocarbon in question is found to be a triplet ground-state. We have occasionally noted specific examples of this phenomenon as we have serendipitously come across them in our previously reported work. ${ }^{[16,25]}$

Such structures have, however, arisen only very occasionally and, even then, they have been associated with somewhat outré molecular graphs ${ }^{[8]}$ that are unlikely to represent the carbon-carbon connectivities ${ }^{[8]}$ of extant or viable conjugated hydrocarbons - examples are $(3,12)$ Coronene $^{[25,26]}$ (structure 1, on the left-hand side of Figure 1) and ${ }^{[16]}$ what Agranat et al. ${ }^{[27]}$ call Corannulene $\left[6^{1}, 6^{1}, 6^{1}, 6^{1}\right.$, $6^{1}, 6^{1}$ ] (structure 2 , on the right-hand side of Figure 1 ).

The problem also arose in some recent calculations (not yet published) in which, following the $a b$ initio calculations of Monaco and Zanasi, ${ }^{[28,29]}$ we attempted to extend our earlier studies of the neutral altans and multiple ('iterated') altans of corannulene and coronene, ${ }^{[30]}$ and their dianions, ${ }^{[31]}$ to the tetra-anions and hexa-anions of the same parent species. 


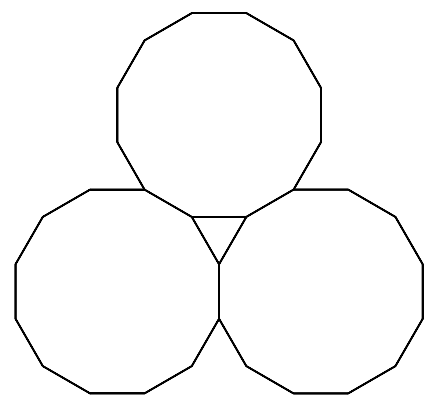

1

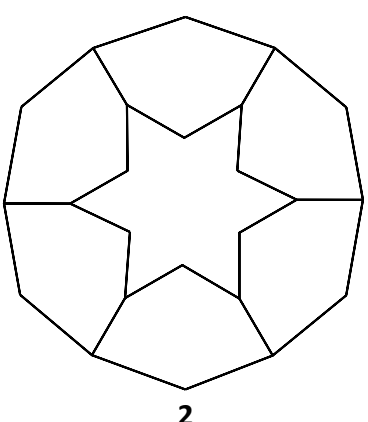

2

Figure 1. Carbon-carbon connectivities ('molecular graphs'[8]) of hypothetical conjugated hydrocarbons $(3,12)$-Coronene ${ }^{[25,26]}$ (1) and Corannulene $\left[6^{1}, 6^{1}, 6^{1}, 6^{1}, 6^{1}, 6^{1}\right],,^{[16,27]}$ latent in both of which is the 'triplet ground-state problem', discussed in the text.

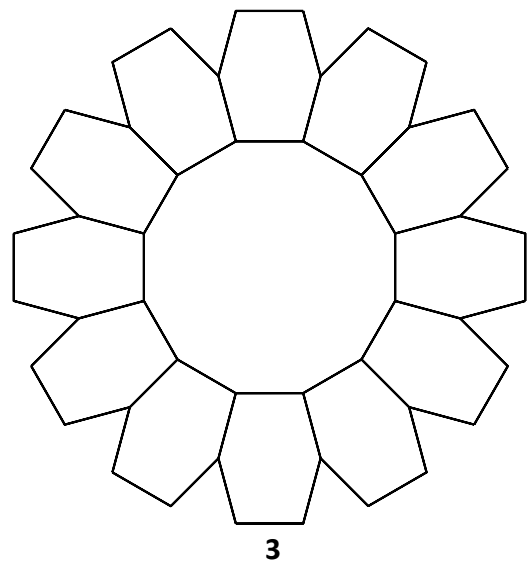

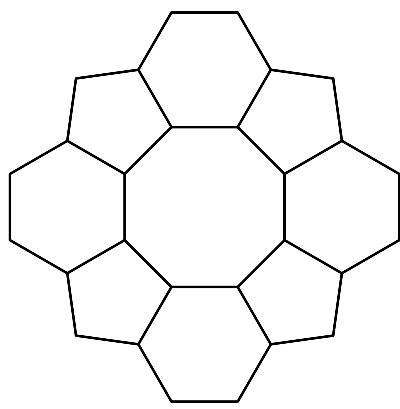

4

Figure 2. Carbon-carbon connectivities ('molecular graphs'[8]) of (left) a hypothetical conjugated hydrocarbon (3) that we have 'designed', and (right) structure 4, the extant [8]-circulene labelled ' 9 ' by Baryshikov et al. in Ref. [32]. The di-anion and the di-cation of $\mathbf{3}$, and the neutral species 4 , are used here in order to illustrate the 'triplet ground-state problem', discussed in the text.

We have also noted this occurrence of what we are somewhat colloquially calling 'the triplet ground-state problem' in some charged species of a hypothetical conjugated system (structure $\mathbf{3}$, on the left-hand side of Figure 2) and in the case of an extant hydrocarbon of the [8]-circulene family (structure 4 , on the right-hand side of Figure 2), the latter of which was recently considered by Baryshikov et al. ${ }^{[32]}$ These two structures will be used in this presentation, along with structures $\mathbf{1}$ and $\mathbf{2}$ (in Figure 1), in order to illustrate the observations that are being documented here about the 'triplet ground-state problem'.

The overall aim of the present communication is to describe the problems that a triplet ground-state brings about, to record some specific examples of this problem's occurrence, and to emphasise that it represents an intrinsic limitation of the HLPM approach. It is, furthermore, also a limitation that is necessarily inherent in other equivalent (though ostensibly different) methods ${ }^{[13-15,33-38]}$ of calculating magnetic properties due to $\pi$-electron ring- currents - methods that are likewise founded on the Hückel molecular-orbital conventions. ${ }^{[11,12,39]}$

\section{THE OVERALL STRUCTURE OF HLPM RING-CURRENT CALCULATIONS}

HLPM 'topological' ring-current and bond-current calculations are carried out in the following four stages: ${ }^{[16-19]}$

(a) Hückel[11,12,39] energy-levels and molecular-orbitals - the latter consisting of appropriate linearcombinations of the atomic orbitals (LCAO) that are centred on each of the carbon atoms - are first calculated from an arbitrarily labelled moleculargraph $^{[8,16-19]}$ representing the carbon-carbon connectivity of the particular conjugated hydrocarbon under study. In the graphtheoretical[8,16-19] language in which the HLPM 
approach $^{[9,10]}$ is frequently cast, these are the eigenvalues and eigenvectors, ${ }^{[11,21]}$ respectively, of the vertex-adjacency matrix ${ }^{[8]}$ of that associated labelled molecular-graph. ${ }^{[8]}$

(b) Then the Aufbau process ${ }^{[20-24]}$ is applied, during the course of which the available $\pi$-electrons are distributed amongst these calculated energy-levels in order to determine a $\pi$-electron ground-state configuration for the hydrocarbon in question. If the Aufbau procedure has been successful - which is not always the case, as is discussed in Refs. [21-23] - then, at this stage, each energy level is known to have been unambiguously assigned either two $\pi$ electrons (in which case that energy level is said to be 'fully occupied' or 'filled'), or just one $\pi$-electron (when it is 'singly occupied'), or no $\pi$-electrons at all (in which latter case the molecular orbital in question is said to be 'unoccupied', or 'empty'). This is all as described on pp. 123-125 of Cotton. ${ }^{[20]}$

(c) Once this $\pi$-electronic ground-state has been established, the $\pi$-electron energy-levels and the LCAO molecular-orbitals (determined in stage (a), above), together with the ground-state $\pi$-electron configuration (determined in (b), above, by invoking the Aufbau process ${ }^{[20]}$ ), are used to calculate certain quantities that are referred to as ${ }^{[16-19]}$ Coulson bondorders ${ }^{[11,40]}$ and self- and mutual imaginary bondbond polarisabilities ${ }^{[15,40]}$ (all of which are explicitly defined in the next section).

(d) These purely graph-theoretical quantities are then combined with geometrical information (in the form of the areas of the various rings within the conjugated system being studied) in order to calculate the HLPM ring-currents and bond-currents.

In the present paper, we shall not be concerned at all with stage (d). We do, however, now consider stages (a)-(c) in more detail.

\section{DETAILS OF STAGES (a)-(c)}

(a) Hückel Energy-Levels and LCAO Molecular-Orbitals The first task in a ring-current calculation carried out by means of the HLPM method is to find the eigenvalues and the corresponding eigenvectors of the vertex-adjacency matrix ${ }^{[8,21]}$ of the molecular graph $^{[8]}$ representing the conjugated hydrocarbon under consideration. If that hydrocarbon has $N$ carbon atoms, then

$$
\left\{\mathbf{c}_{J}\right\}_{J=1,2, \ldots, N}\left(=\left\{\left(c_{J 1} c_{J 2} \ldots c_{J N}\right)^{T}\right\}_{J=1,2, \ldots, N}\right)
$$

denotes the set of eigenvectors (i.e., the standard, field- free Hückel coefficients in each of the N LCAO molecularorbitals), and $\left\{E_{J}\right\}_{J=1,2, N}$ denotes the family of eigenvalues of that molecular graph - that is, they are the Hückel energy-levels, $\varepsilon_{\jmath}$, when expressed in the conventional form $\varepsilon_{j}=\alpha+E_{j} \beta$, where $\alpha$ and $\beta$ are the standard Hückel Coulomb-integrals and resonanceintegrals, respectively. ${ }^{[11,12,39]}$

It is assumed in the above that the eigenvectors are (a) normalised and (b) orthogonalised (if degeneracies amongst the energy levels - that is, repetitions within the eigenvalues - make this latter operation necessary because the eigenvectors of the real-symmetric vertexadjacency matrix are then no longer all automatically mutually orthogonal). This matter is gone into in considerable detail on pp. 63-65 of Ref. [21].

\section{(b) The Aufbau Process and the Possibility of a Triplet Ground-State}

Once the energy levels are established, as in stage (a), above, they may notionally be thought of as being arranged vertically in (from the bottom to the top) increasing order of energy, and the available $\pi$-electrons ( $N$ for a neutral species, $(N+2)$ for a di-anion, $(N-2)$ for a di-cation, etc.) are then assigned to occupy the energy-levels, beginning with the one of lowest energy, according to the well-known Aufbauprinzip, which incorporates the Pauli Exclusion Principle and Hund's Rules of Maximum Multiplicity (e.g., Ref. [20]). This process yields a family of $N$ numbers, $\left\{v_{J}\right\}_{J=1,2, \ldots, N}$ (which are the occupations numbers assigned, by the Aufbau process, to the energy levels/eigenvalues); the elements of the family $\left\{v_{J}\right\}_{J=1,2, N}$ are all either 0,1 or 2 .

Rouvray and one of the present authors (RBM) ${ }^{[22,23]}$ showed many years ago that the Aufbau process may effectively be simulated by means of an algorithm, the outcome of which depends only on the relative order of the eigenvalues/energy levels computed (as in (a), above) for the structure. In that sense, therefore, since the order of the energy levels/eigenvalues - being latent, as they are, in the vertex-adjacency matrix - depends only on the carbon-atom connectivity in the molecular graph in question, the Aufbau procedure ${ }^{[20]}$ may itself be thought of as an entirely graph-theoretical process. (Please see Refs. [21-23] for full details of this idea.)

Now, our purpose in this communication is to point out that there are occasions - after an attempt is made by application of the Aufbau Process[ ${ }^{20-23]}$ to determine a $\pi$ electronic ground-state configuration - when that groundstate turns out to be a triplet, in which the highest-occupied levels are doubly degenerate and each is singly occupied (i.e, $v_{\jmath}=1$ for both of those degenerate energy-levels). This case arises when the last two $\pi$-electrons to be distributed in the course of applying the Aufbau process ${ }^{[20-23]}$ have to be assigned to a pair of doubly degenerate 
molecular-orbitals (that is, a pair of repeated eigenvalues of the molecular graph ${ }^{[8,17,21]}$ ) - thereby necessarily resulting in two unpaired spins and thus a triplet groundstate. This is where there is a problem with the HLPM approach - a problem that is shared with other ostensibly different formalisms ${ }^{[33-38]}$ that also rely on the simple Hückel model. ${ }^{[11,12,39]}$ How this comes about is explained in the next sub-section.

A referee has pointed out that there are wider and more sophisticated considerations concerning the Aufbau process than are addressed here - see, for example, Refs. [41-43] - which involve predicting spin by use of a variety of different techniques. In this work, however, we are avowedly using Hückel Theory only. We are also strictly adhering to Hund's rule ${ }^{[20]}$. Consideration of species such as extended graphite and graphene-like structures - together with concomitant boundary-conditions - is, therefore, not directly relevant to our work here on discrete molecules. Furthermore, neither are we doing configuration interaction nor valance bond calculations. We are merely establishing the circumstances under which application of the HLPM approach, in its originally proposed form,,$[9,10]$ exposes certain pathological cases. In addition, it should be noted that he HLPM formalism is explicitly defined ${ }^{[9,10,17]}$ to incorporate the graph-theoretical algorithm of Refs [17, 21-23] in order to mimic the conventional Aufbau process. ${ }^{[20]}$ That is why no other form of Aufbau scheme is entertained here.

\section{(c) Coulson Bond-Orders and Self- and Mutual Bond- Bond Polarisabilities}

The third stage in the HLPM approach is to take the graphtheoretical data - the eigenvalues and the eigenvectors of the vertex-adjacency matrix of the molecular graph - that have been obtained in stage (a), above, and the groundstate $\pi$-electronic configuration - the family of eigenvalue/energy-level occupation-numbers, $\left\{v_{J}\right\}_{J=1,2, \ldots, N}$ - established by an application of the Aufbau process in stage (b), in order to calculate two types of graphtheoretical Hückel molecular-orbital indices that are needed in the ring-current calculations. ${ }^{[17]}$ These are the well-known Coulson bond-order, ${ }^{[11,12,40]} P_{(r s)}$, for the bond between carbon atoms $r$ and $s$ (defined in equation (1), below) and the imaginary mutual- (and self-) bond-bond polarisabilities, $\bar{\pi}_{(r s)(t u)}$ and $\bar{\pi}_{(r s)(r s)}$, respectively, originally defined by McWeeny in Ref. [15] (with some misprints that are corrected in the Appendix of Ref. [40]). The imaginary mutual bond-bond polarisabilities, $\bar{\pi}_{(r s)(t u)}$, between bonds $(r s)$ and $(t u)$, are defined in equations (2) and (3), below; the imaginary self bond-bond polarisability for the bond $(r s)$ is obtained by replacing ' $t u$ ' everywhere with ' $r s$ ' in equations (2) and (3). All the symbols in equations (1) - (3) have been defined in the previous sub-section, with the exception of $M$, which is the number of doubly-occupied orbitals. It should be emphasised that, for equations (2) and (3) to be valid, there must be no singly-occupied orbitals; the number of unoccupied orbitals is thus $(N-M)$

$P_{(r s)}=\sum_{J=1}^{N} v_{J} c_{J r} c_{J s}$

$\bar{\pi}_{(r s)(t u)}=\pi_{r s, t u}-\pi_{r s, u t}+\pi_{s r, u t}-\pi_{s r, t u}$,

where

$\pi_{r s, t u}=\frac{2}{\beta} \sum_{l=1}^{M} \sum_{J=M+1}^{N} \frac{c_{l r} c_{J s} c_{J t} c_{l u}}{E_{l}-E_{J}}$.

The actual fine details of the calculations - such as equations (1)-(3), above - are, however, not necessary for the present considerations and, for any interested reader, they may be found in our earlier reviews ${ }^{[16-19]}$; the crucial points that we emphasise here are that

(i) Bond order is defined for open-shell states - see pp. 63-64 of Ref. [11]: some of the $\left\{v_{J}\right\}_{J=1,2, \ldots, N}$ in equation (1) may thus be equal to 1 for certain values of the running-index, $J$.

(ii) The imaginary bond-bond polarisabilities, $\bar{\pi}_{(r s)(t u)}$, by contrast, are defined (by equations (2) and (3)) only for closed-shell ground-states - that is, only when every energy level is either unoccupied or doubly occupied. In particular, we observe that, in equation (3), $M$ denotes the number of doubly-occupied orbitals and the other $(N-M)$ orbitals are all unoccupied; there must be no singly occupied orbitals.

Triplet ground-states are, accordingly, not catered for in the HLPM method, nor are they by other methods ${ }^{[33-38]}$ that are based on the Hückel approach. As a very special case of the more-general scenario outlined here, the first explicit encounter with this phenomenon was in the context of the [4n]-annulenes without 'bond-alternation', studied by Pople and Untch ${ }^{[44]}$ in their classic paper of more than fifty years ago. Consequently, in these 'triplet ground-state' situations, infinite paramagnetism is formally to be predicted - as was pointed out originally by Pople and Untch, ${ }^{[44]}$ was re-iterated and further discussed in Note 21 on Page 170 of Ref. [11], and as was also more recently re-emphasised by Aihara. ${ }^{[45]}$

\section{EXAMPLES OF CASES IN POINT}

(i) Structure 1 (shown on the left-hand side of Figure 1) The left-hand section of Scheme 1, labelled ' $\mathrm{I}$ ', shows the energy levels (eigenvalues) for the neutral species of structure 1 (which is depicted on the left-hand side of Figure 1). They are presented according to the conventions explained in the caption to Scheme 1. 

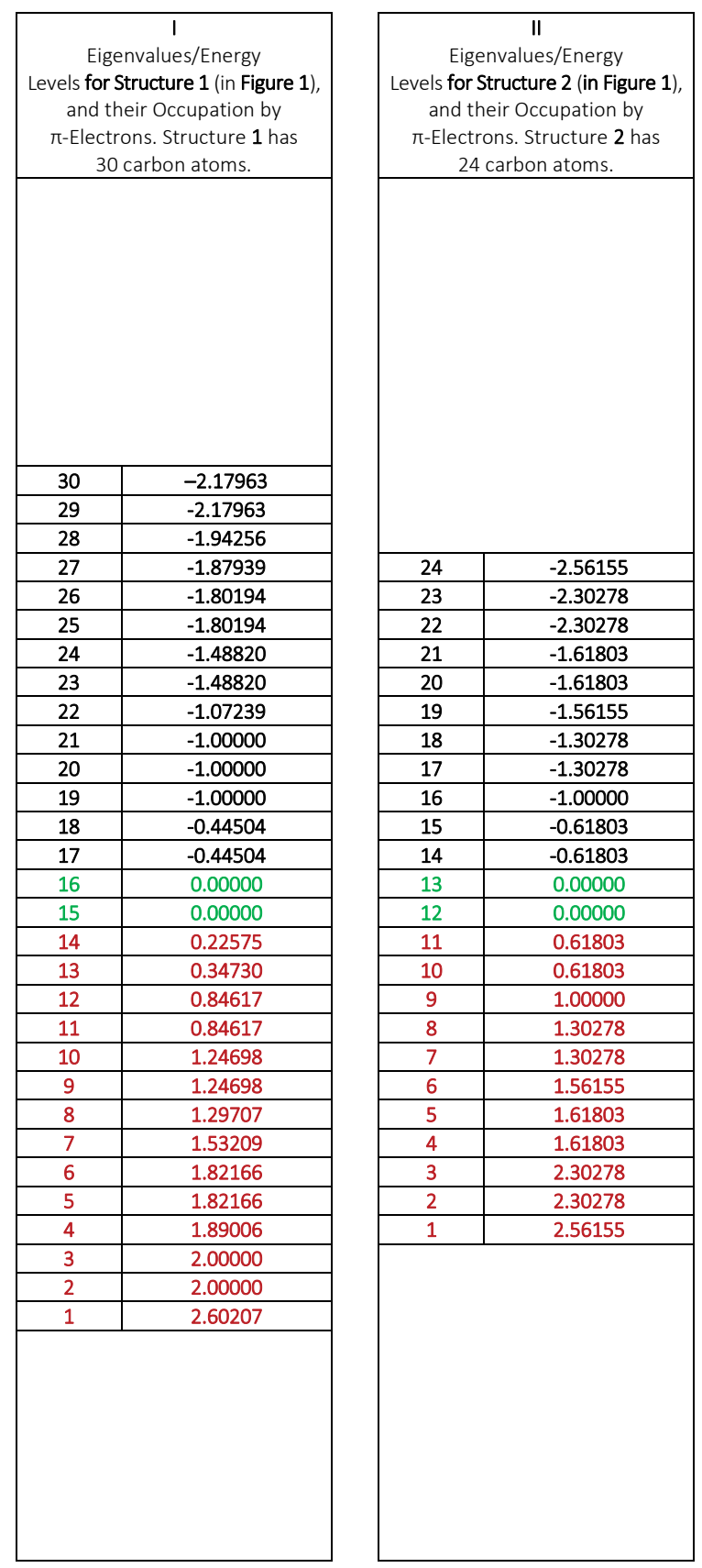

\begin{tabular}{|c|c|}
\hline \multicolumn{2}{|c|}{$\begin{array}{c}\text { III } \\
\text { Eigenvalues/Energy } \\
\text { Levels for Structure } 3 \text { (in Figure 2), } \\
\text { and their Occupation by } \\
\pi \text {-Electrons. Structure } 3 \text { has } \\
48 \text { carbon atoms }\end{array}$} \\
\hline 48 & -2.67513 \\
\hline 47 & -2.52434 \\
\hline 46 & -2.52434 \\
\hline 45 & -2.21432 \\
\hline 44 & -2.21432 \\
\hline 43 & -1.93185 \\
\hline 42 & -1.93185 \\
\hline 41 & -1.67513 \\
\hline 40 & -1.67513 \\
\hline 39 & -1.53919 \\
\hline 38 & -1.41421 \\
\hline 37 & -1.41421 \\
\hline 36 & -1.41421 \\
\hline 35 & -1.41421 \\
\hline 34 & -1.21432 \\
\hline 33 & -1.00000 \\
\hline 32 & -1.00000 \\
\hline 31 & -1.00000 \\
\hline 30 & -0.79229 \\
\hline 29 & -0.79229 \\
\hline 28 & -0.53919 \\
\hline 27 & -0.53919 \\
\hline 26 & -0.51764 \\
\hline 25 & -0.51764 \\
\hline 24 & 0.51764 \\
\hline 23 & 0.51764 \\
\hline 22 & 0.53919 \\
\hline 21 & 0.53919 \\
\hline 20 & 0.79229 \\
\hline 19 & 0.79229 \\
\hline 18 & 1.00000 \\
\hline 17 & 1.00000 \\
\hline 16 & 1.00000 \\
\hline 15 & 1.21432 \\
\hline 14 & 1.41421 \\
\hline 13 & 1.41421 \\
\hline 12 & 1.41421 \\
\hline 11 & 1.41421 \\
\hline 10 & 1.53919 \\
\hline 9 & 1.67513 \\
\hline 8 & 1.67513 \\
\hline 7 & 1.93185 \\
\hline 6 & 1.93185 \\
\hline 5 & 2.21432 \\
\hline 4 & 2.21432 \\
\hline 3 & 2.52434 \\
\hline 2 & 2.52434 \\
\hline 1 & 2.67513 \\
\hline
\end{tabular}

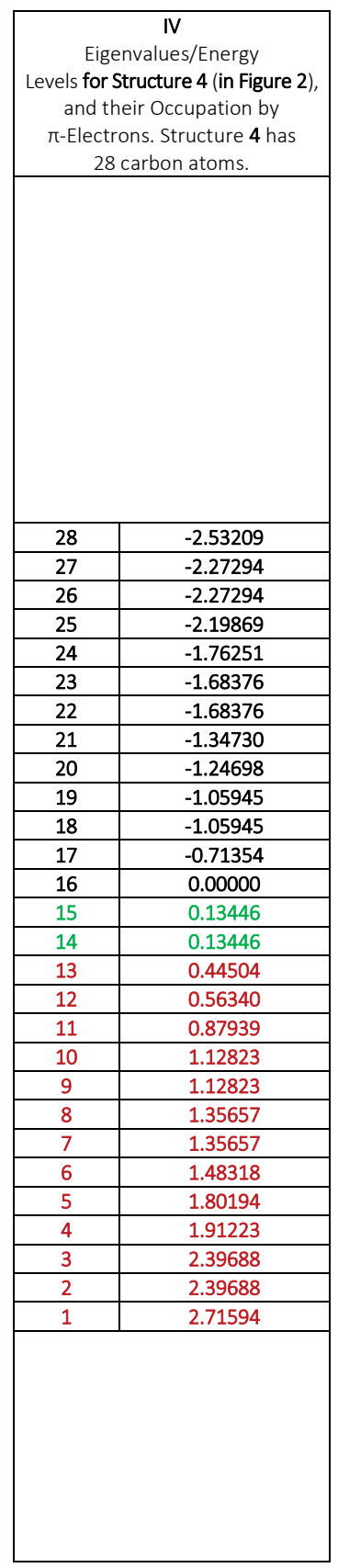

Scheme 1. Disposition of Eigenvalues/Energy-Levels for structures 1 and 2 (in Figure 1) (in the sections headed ' $\mathrm{I}$ ' and ' $\mathrm{I}$ ', respectively) and for structures 3 and $\mathbf{4}$ (in Figure 2) (in the sections headed 'III' and 'IV', respectively), together with their corresponding occupation by $\pi$-electrons after the Aufbauprinzip has been applied. Colour Scheme: Doubly ('fully') occupied orbitals are depicted in red, singly occupied orbitals are presented in green, and unoccupied ('empty') orbitals are denoted in black. In each of the four sets of data - headed by the labels ' $I$ ', ' $I$ ', 'III' and 'IV' - the integer in the left-hand column of the pair of columns pertaining to each of the four structures considered (1-4) is the molecular-orbital labelling (' 1 ' being the lowest-energy bonding-orbital and ' 30 ', '24', ' 48 ' and ' 28 ' being the highest-energy anti-bonding orbitals in, respectively, structures 1, 2, 3 and 4). In the right-hand column of each pair appropriate to the four sets (I-IV) of data pertaining to each of the structures $1-4$ is a floating-point number; this is the quantity $k$ when the Hückel molecular-orbital energy is represented in the conventional way as $\alpha+k \beta$ (with being $\alpha$ and $\beta$ being, respectively, the standard Hückel Coulomb-integral and the standard Hückel resonance-integra|11,12,39). 
After an application of the Aufbau process to the neutral species of structure $\mathbf{1}-$ in the course of which 30 $\pi$-electrons are notionally to be assigned to the energy levels - the first 28 electrons go into the orbitals (labelled 1-14) coloured in red in the section of Scheme 1 labelled 'I'; each of these orbitals is therefore fully occupied with two electrons. The last two electrons of the 30 to be distributed are then available to be placed, with paired spins, in the non-bonding energy-level 15 . However, level 16 is also a non-bonding orbital; according, therefore, to the rules of the Aufbau procedure, the two non-bonding orbitals (15 and 16, coloured in green in section I of Scheme 1) are consequently both singly occupied. The remaining (antibonding) orbitals - which are denoted in black in section I of Scheme 1 and are labelled 17-30 - are unoccupied. The result is a triplet $\pi$-electronic ground-state for the neutral species of structure 1. (It should be noted, however, that, for example, the di-anion (12-) of structure 1 would have a closed-shell ground-state, as the two extra $\pi$-electrons that it possesses would be accommodated in levels 15 and 16, thereby completing the double occupation of both of these two non-bonding orbitals, and thus resulting in a closedshell structure.)

(ii) Structure 2 (shown on the right-hand side of Figure 1) The section of Scheme 1 headed 'II' shows the energy levels (eigenvalues) for the neutral species of structure $\mathbf{2}$ (depicted on the right-hand-hand side of Figure 1). They are likewise presented according to the conventions explained in the caption to Scheme 1. It should be noted that, in accordance with the Coulson-Rushbrooke Theorem (please see Chapter 6, pp. 88-110, and Appendix D, pp. 159-166, of Ref. [11]), the eigenvalues of the bipartite graph $^{[8,11]}$ that represents structure $\mathbf{2}$ are seen to occur in \pm pairs, around zero.

After an application of the Aufbau process, in which $24 \pi$-electrons are notionally to be distributed, the first 22 electrons go into the bonding orbitals (labelled 1-11) coloured in red in section II of Scheme 1 and each of these is therefore fully occupied with two electrons. The last two electrons are then available to be assigned to the nonbonding energy-level 12 . However, in an exact analogy with what happened in the case of structure 1 in Scheme 1, level 13 in section II of Scheme 1 is also a non-bonding orbital; according, therefore, to the rules of the Aufbau procedure, the two non-bonding orbitals (12 and 13, coloured in green in section II of Scheme 1) are, as a result, singly occupied. The remaining orbitals - namely, the anti-bonding orbitals, denoted in black in section II of Scheme 1 and labelled 14$24-$ are all unoccupied. The result is once again a triplet $\pi$-electronic ground-state for the neutral species of structure 2. (It should be noted that - as was the case with structure $\mathbf{1}$ - the di-anion $\left(\mathbf{2}^{2}\right)$ of structure $\mathbf{2}$ would have a closed-shell ground-state, as the two extra $\pi$-electrons that it possesses would complete the full occupation of the two non-bonding orbitals 12 and 13.)

\section{(iii) Structure 3 (shown on the left-hand side of Figure 2)}

The section of Scheme 1 headed 'III' presents the energy levels (eigenvalues) for the neutral species of the 'designed' structure $\mathbf{3}$ (shown on the left-hand side of Figure 2). They are likewise displayed according to the conventions explained in the caption to Scheme 1. Once again, as with structure 2 , the eigenvalues of the bipartite graph ${ }^{[8,11]}$ that represents structure 3 occur in \pm pairs, around zero (in accordance with the Coulson-Rushbrooke Theorem, ${ }^{[11]}$ referred to above).

After an application of the Aufbau process, in which $48 \pi$-electrons are notionally to be distributed, all 48 may straight away be accommodated in the (bonding) energy levels labelled 1-24 and coloured in red in the section of Scheme 1 headed 'III'; each of these orbitals is therefore fully occupied with two electrons. The remaining orbitals - which are anti-bonding ones and which are denoted in black in Scheme 1 and are labelled 25-48 - are unoccupied. The result is - in contrast to what occurred in the case of the neutral species $\mathbf{1}$ and $\mathbf{2}-$ a closed-shell $\pi$ electronic ground-state for the neutral species of structure 3. This means that, by use of the standard HLPM procedures (of which full details are given in, for example, Refs. [9,10,16-19]), 'topological' ring-currents and bondcurrents may be computed for the neutral species of structure $\mathbf{3}$ (which is illustrated on the left-hand side of Figure 2). For the record, we note that the calculated ringcurrent intensities (expressed as a ratio to the benzene value) are $-0.161-$ the negative sign indicates that the ring current is paramagnetic - for the inner, 12-membered ring and +1.113 (diamagnetic) for each of the 12 peripheral six-membered rings. As for the bond currents, a current of magnitude 1.113 flows around the perimeter (of length 36 $=$ ' $[4 n]$ ', with $n=9$ ), in the anti-clockwise (diamagnetic) direction, and one of magnitude 1.274 flows around the inner, 12-membered ring (' $[4 n]$ ', with $n=3$ ), in the clockwise (paramagnetic) sense. By symmetry, the ringcurrent intensity in all the 'spokes' bonds, ${ }^{[46,47]}$ connecting the inner ring to the perimeter, is precisely zero.

However, consultation of section III of Scheme 1 shows that (for example) the dianion $\left(\mathbf{3}^{2-}\right)$ of structure 3 would again have a triplet ground-state, because the 'extra' two electrons that the di-anion possess - but which the neutral species does not possess - would have to be assigned, by the Aufbau process, so that one was accommodated by level 25 and the other by the degenerate level 26, as both anti-bonding levels 25 and 26 are of energy $(\alpha-0.51764 \beta)$. A similar situation would be encountered on considering the di-cation $\left(3^{2+}\right)$ of 3 . This has 
two fewer electrons than does the neutral $\mathbf{3}$ and so, with only 46 electrons now to be assigned when the Aufbau process is invoked, 44 of them would be accommodated by double occupation in each of levels $1-22$, but the last two would have to sit, each on its own, in the two degenerate bonding-levels 23 and 24, which are both of energy $(\alpha+$ $0.51764 \beta$ ). Hence, a triplet ground-state would once again be encountered.

(iv) Structure 4 (shown on the right-hand side of Figure 2) Finally, the section of Scheme 1 headed 'IV' shows the energy levels (eigenvalues) for the neutral species of structure $\mathbf{4}$ (shown on the right-hand side of Figure 2). They are likewise displayed according to the conventions explained in the caption to Scheme 1. This extant structure (4), along with some doubly-charged species related to it, was recently discussed by Baryshikov et al. ${ }^{[32]}$

During an application of the Aufbau process, in which $28 \pi$-electrons are notionally to be distributed, the first 26 electrons conveniently go into the bonding orbitals (labelled 1-13) that are coloured in red in the section of Scheme 1 headed 'IV'; each of these orbitals is therefore fully occupied with two electrons. The last two electrons are then available to be assigned to the energy-level labelled 14 (also a bonding orbital). However, by analogy with what happened with structure 1 in section I of Scheme 1 and with structure $\mathbf{2}$ in section II of Scheme 1, this level 14 in section IV of Scheme 1 is degenerate with level 15, both bonding orbitals having energy $(\alpha+0.13446 \beta)$. According, therefore, to the rules of the Aufbau procedure, these two degenerate bonding-orbitals orbitals (14 and 15), coloured in green in section IV of Scheme 1, are, as a result, singly occupied. The remaining orbitals - one (labelled 16) which is non-bonding and the rest (labelled 17-28) which are all anti-bonding - are denoted in black in section IV of Scheme 1 and are all unoccupied. The result is once again a triplet $\pi$-electronic ground-state for the neutral species of structure 4.

It should be noted that - as was the case with, for example, structure $\mathbf{1}-$ the dianion $\left(4^{2-}\right)$ of structure 4 would have a closed-shell ground-state, as the two extra $\pi$ electrons that it possesses when compared with the neutral species would completely fill the two bonding orbitals labelled 14 and 15, each of which, in neutral structure 4 itself, is only singly occupied, as is evident from section IV of Scheme 1. The topological ring-currents and bondcurrents of $4^{2-}$ can, therefore, be computed, by the standard methods described in detail in our previous reviews. ${ }^{[16-19]}$ Since this charged species is an extant structure recently discussed in Ref. [32], we take the opportunity to present, in Figure 3, the topological HLPM ring-currents and bond-currents of the di-anion of structure 4. For the full details of how these bond currents

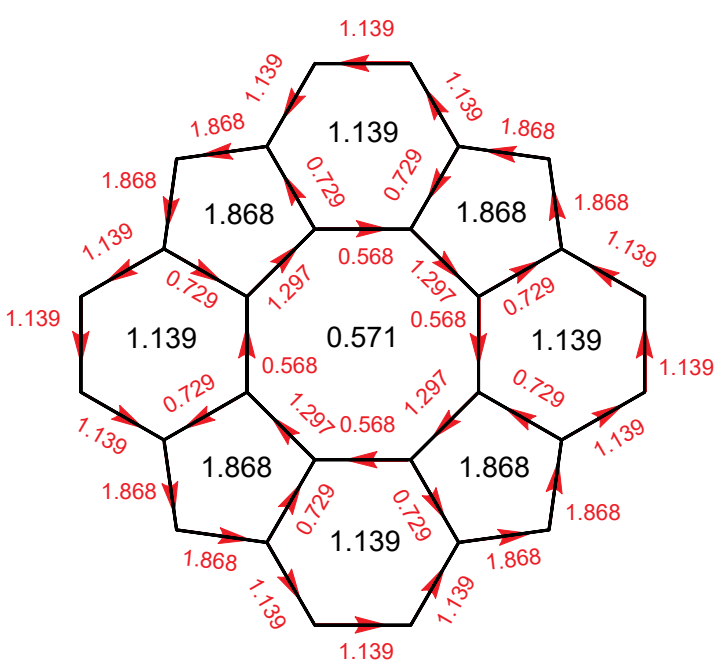

Figure 3. HLPM ring-current and bond-current maps for $4^{2-}$, the di-anion of structure 4 (introduced in Ref. [32]). Figures in black in the centres of rings denote the corresponding (diamagnetic) ring-current intensities (expressed as a ratio to the benzene value), whilst red figures written along the bonds denote the bond-currents (in the directions indicated by the arrows) that are consistent with them

and ring currents were computed, the reader is referred to extensive and fully-worked examples in two reviews ${ }^{[16,17]}$ and to several briefer, but still substantial, examples presented in Refs. [9,18 and 19].

All ring-currents in $\mathbf{4}^{2-}$ are diamagnetic; bond currents are such that there is a diamagnetic flow (that is, in the anti-clockwise direction) around the perimeter, which is of length $20(=[4 n]$, with $n=5)$, and a flow in the clockwise (paramagnetic) sense around the inner 8membered ring (which is [4n], with $n=2$ ). $\ln 4^{2-}$, the spokes bonds ${ }^{[46,47]}$ connecting the inner (8-membered) ring to the 20-membered perimeter do not (as, by contrast, was the case with the spokes bonds of structure 3 ) carry zero current by symmetry but, as can be seen from Figure 3 , they do in fact bear a substantial current - some $73 \%$ of the benzene value.

In Figure 3, the numbers in black in the centres of the rings are the corresponding topological ring-currents (expressed as a ratio to the benzene value). The positive nature of all of these ring currents indicates, on the convention being used, that they are diamagnetic and thus are considered to circulate in the anti-clockwise direction around their respective rings. Figures written in red along the bonds denote the corresponding HLPM bond-currents (which, again, are expressed as a ratio to the corresponding bond-current calculated, by the same method, for benzene). The arrow pointing along each bond indicates the direction of flow of the bond current in that bond. By 
virtue of the validity of Kirchhoff's Law of Conservation of Currents at Junctions, ${ }^{[6]}$ all bond-currents - the quantities depicted in red in Figure 3 - are automatically predetermined once the ring currents in all rings - the quantities in black, in Figure 3 - have been specified. [48-50] As we have previously pointed out, $[17,18,50]$ the strict observance of the Kirchhoff Conservation Law in the case of the HLPM approach is essentially because, on the assumptions made, a conjugated hydrocarbon really is mimicked as if it were a microscopic version of a standard macroscopic Kirchhoff network, with EMFs and 'arms' consisting of macroscopic wires, loop currents (the macroscopic analogy of ring currents) around the closed regions, and currents (the macroscopic analogy of bond currents) in the individual wires. Conservation of currents at junctions in ab-initio calculations is, by contrast, strictly observed only in the limit of an infinite basis set. ${ }^{[51-54]}$

\section{ARTIFICIALLY OBVIATING THE TRIPLET GROUND-STATE PROBLEM BY DELIBERATELY BREAKING SYMMETRY}

A referee has pointed out that a renowned example of a system for which Hückel molecular-orbital (HMO) theory predicts a triplet ground state is square cyclobutadiene, with all four resonance integrals, $\beta$, being the same. In this case, the non-bonding energy-level is doubly degenerate and this, of course, is what gives rise to the triplet groundstate - see, for example, Page 78 of Ref. [11]. Another well-known example is cyclooctatetraene, the HMO description of which behaves in very much the same way, although, in practice, the most important aspect of this structure is that, on steric grounds, it is severely nonplanar; (please see Page 81 of Ref. [11]). In addition, we have ourselves already drawn attention in the present paper to an analogous situation that obtains in the case of the [4n]-annulenes; (please see Ref. [44] and Note 21 on Page 170 of Ref. [11]). However, as the above-mentioned referee has reminded, if the resonance integrals - in, say, cyclobutadiene - are allowed to alternate as $\beta(s)$ and $\beta(I)$, mimicking 'short' and 'long' bonds, respectively, this degeneracy disappears by means of a pseudo JahnTeller/Renner distortion to a rectangular shape (as described by Coulson et al. on Pages 79-80 and in Note 13 on Page 172 of Ref. [11]); as a result, the total $\pi$-electron energy goes down. This has led to the referee's suggestion of investigating whether the examples considered in this paper in which a triplet ground-state is encountered might not behave in a similar way, if given the opportunity to lower the symmetry of the problem by the introduction of alternation in the values of the resonance integrals. Returning again to our structure $\mathbf{2}$, illustrated on the righthand side of Figure 1, we have taken up the referee's suggestion and (somewhat arbitrarily) we have effected the following changes:

(a) For those bonds around the periphery the resonance integrals have been changed so that they alternate between 0.90 and 1.10 (in units of $\beta$ ).

(b) For those bonds forming the 'inner star', the resonance integrals alternate between 0.85 and 1.15.

(c) For the 'spokes bonds', connecting the inner star to the periphery, the resonance integrals have been left at 1.00 .

This results in the ordered eigenvalue/energy-level list depicted in Scheme 2 (with the same colour conventions as in Scheme 1: red indicates doubly-occupied orbitals and black denotes empty energy-levels).

This, therefore, is seen to result in a closed-shell ground-state, as desired. This situation is to be compared with column II of Scheme 1, which shows the outcome of the

\begin{tabular}{|c|c|}
\hline \multicolumn{2}{|c|}{$\begin{array}{l}\text { Eigenvalues/Energy } \\
\text { Levels for Structure } 2 \text { (in Figure } 1 \text { ) } \\
\text { when alternating values are assigned } \\
\text { to Resonance Integrals (as described in } \\
\text { the text), and the Occupation of those } \\
\text { Levels by } \pi \text {-Electrons. Structure } 2 \text { has } \\
24 \text { carbon atoms. }\end{array}$} \\
\hline 24 & -2.5615528 \\
\hline 23 & -2.3072793 \\
\hline 22 & -2.3072793 \\
\hline 21 & -1.6396742 \\
\hline 20 & -1.6396742 \\
\hline 19 & -1.5615528 \\
\hline 18 & -1.3072728 \\
\hline 17 & -1.3072728 \\
\hline 16 & -1.0615108 \\
\hline 15 & -0.6395066 \\
\hline 14 & -0.6395066 \\
\hline 13 & -0.0565232 \\
\hline 12 & 0.0565232 \\
\hline 11 & 0.6395066 \\
\hline 10 & 0.6395066 \\
\hline 9 & 1.0615108 \\
\hline 8 & 1.3072728 \\
\hline 7 & 1.3072728 \\
\hline 6 & 1.5615528 \\
\hline 5 & 1.6396742 \\
\hline 4 & 1.6396742 \\
\hline 3 & 2.3072793 \\
\hline 2 & 2.3072793 \\
\hline 1 & 2.5615528 \\
\hline
\end{tabular}

Scheme 2. Disposition of Eigenvalues/Energy-Levels for Structure 2 (in Figure 1) together with their corresponding occupation by $\pi$-electrons after the Aufbauprinzip has been applied. The Colour Convention is as in Scheme 1: Doubly ('fully') occupied orbitals are depicted in red, and unoccupied ('empty') orbitals are denoted in black. 
original HLPM calculation on structure $\mathbf{2}$, when all resonance integrals were assigned the standard value, $1.00 \beta$.

Even though the quantifying of resonance integrals in the above calculation was subjective and arbitrary, this exercise - suggested by the referee - does allow the claim that there exists at least one set of resonance-integral values that has the effect of splitting the degeneracies of the non-bonding levels labelled 12 and 13 in column II of Scheme 1. In this case, these divide into two new levels likewise labelled, in Scheme 2, as 12 and 13 - of energies $(\alpha+0.0565232 \beta)$ and $(\alpha-0.0565232 \beta)$, respectively. This splitting of the two previously degenerate non-bonding levels into a bonding orbital and a symmetrically paired anti-bonding one thus enables a 'closed-shell ground-state' to be established. This state of affairs thereby obviates the triplet ground-state problem - at least in the case of this particular example.

We noted earlier that structure $\mathbf{2}$ is an alternant species (that is, one whose carbon-carbon connectivity is represented by a bipartite molecular-graph), $[8,11,21]$ and that the 'pairing' of eigenvalues prescribed by the CoulsonRushbrooke Theorem (discussed at length in Chapter 6 and Appendix D of Ref. [11]) is, accordingly, displayed in column II of Scheme 1 (as was observed earlier) and in Scheme 2. It may be noted that Schemes 1 and 2 are also in accord with the observation that the Coulson-Rushbrooke Theorem applies irrespective of the values assigned to the resonance integrals; (this point is discussed on Page 164 of Ref. [11]). Most eigenvalues (though not all, as this example shows) do change when the resonance integrals are changed, but all eigenvalues - whatever their value - continue to comply with the provisions of the Coulson-Rushbrooke Theorem and are, accordingly, still 'paired', as Scheme 2 shows in this particular example of structure $\mathbf{2}$.

In summary, although this excursion into variation of resonance integrals has been instructive, it should be emphasised that not having all resonance integrals set at the standard value of $1.00 \beta$ contravenes the 'rules' of the HLPM approach, as defined in Refs. [9 and 10]. That is why, in this work - as in our other recent applications of the rudimentary, pseudo graph-theoretical, HLPM approach resonance integrals have not generally been varied from the standard value of $1.00 \beta$.

\section{CONCLUSION}

In this communication we have drawn attention to a difficulty that arises in the Hückel-London-PopleMcWeeny approach to calculating topological ring-currents and bond-currents in conjugated hydrocarbons. This happens whenever application of the Aufbau process to the eigenvalues/energy levels of the vertex-adjacency matrix of the hydrocarbon's molecular graph results in a triplet $\pi$-electronic ground-state for the system. This difficulty has been illustrated by explicit appeal to four of only about half a dozen examples that the authors, over the years, have come across in the course of carrying out HLPM calculations on scores of different conjugated systems. Not only are instances of this phenomenon apparently rare but, even when they do arise, they have tended to be associated with molecular graphs that are unlikely to represent the carboncarbon connectivities of existing or potentially viable conjugated hydrocarbons. Of the structures giving rise to the problem that have been dealt with here, for example, only one (structure 4) is, to our knowledge, an extant species.

Despite its ostensibly infrequent occurrence, we nevertheless emphasise that this 'triplet ground-state problem' does represent an intrinsic limitation of the HLPM approach. It is, furthermore, also a limitation that is necessarily inherent in other equivalent (though ostensibly different) methods[13-15,33-38] of calculating magnetic properties due to $\pi$-electron ring-currents - methods that are likewise founded on the Hückel molecular-orbital conventions. ${ }^{[11,12,39]}$ When a triplet ground-state arises, topological ring-currents and bond-currents cannot be calculated by the HLPM method, ${ }^{[9,10,13-19]}$ and its equivalents. ${ }^{[33-38]}$ Infinite paramagnetism is formally to be predicted in such situations. ${ }^{[11,44,45]}$

We conclude by drawing attention to an observation that Rouvray and one of the present authors (RBM) made in Refs. [22] and [23] and which was further discussed in Ref. [21]: it was shown in those places that the Aufbau process may effectively be simulated by an entirely graphtheoretical algorithm and that, for certain bizarre graphs that are unlikely to represent the carbon-carbon connectivity of viable molecules, no unambiguously defined $\pi$-electronic ground-state of any sort - let alone a closed-shell one - can be devised when the Aufbau process is applied. ${ }^{[21-23]}$ It is, of course, self-evident that HLPM ring-currents cannot be computed for the network in question in these cases, either.

Acknwledgements. One of us (RBM) would like to pay tribute to the honorand of MATH/CHEM/COMP 31, the late Edward Kirby, for his warm friendship over a period of some forty years, and for the privilege of collaborating with him for a quarter of a century. Both authors of the present paper appreciate very helpful discussions and correspondence with Mr. C. W. Haigh on the question of the conditions under which equation (3) is valid. We are glad to have received some very encouraging comments and helpful suggestions from referees, one of which resulted in our adding the penultimate section of the paper. RBM is very grateful to the Master and Fellows of Peterhouse, Cambridge for his election as a Visiting Fellow of the College during the Easter Term, 2018. 


\section{REFERENCES}

[1] E. C. Kirby, R. B. Mallion, P. Pollak, J. Chem. Soc. Faraday Trans. 1993, 89, 1945-1953. https://doi.org/10.1039/ft9938901945

[2] E. C. Kirby, R. B. Mallion, P. Pollak, Mol. Phys. 1994 83, 599-602.

https://doi.org/10.1080/00268979400101461

[3] E. C. Kirby, D. J. Klein, R. B. Mallion, P. Pollak, H. Sachs, Croat. Chem. Acta 2004, 77, 263-278. https://hrcak.srce.hr/102674

[4] E. C. Kirby, R. B. Mallion, P. Pollak, 'Counting spanning trees in toroidal Fullerenes', in: F. Cataldo, P. Milani, (Series Editors.), F. Cataldo, A. Graovac, O. Ori, (Volume Editors.), Sir Harold Kroto, (Foreword), Carbon Materials: Chemistry and Physics Volume 4: The Mathematics and Topology of Fullerenes, Springer, Dordrecht, Heidelberg, London \& New York, 2010, Chapter 10, pp. 187-204. https://doi.org/10.1007/978-94-007-0221-9_10

[5] E. C. Kirby, R. B. Mallion, P. Pollak, P. J. Skrzyński, Int. J. Chem. Model. 2015, 6, 255-274.

[6] E. C. Kirby, R. B. Mallion, P. Pollak, P. J. Skrzyński, Croat. Chem. Acta 2016, 89, 403-417. https://doi.org/10.5562/cca2995

[7] E. C. Kirby, R. B. Mallion, P. Pollak, P. J. Skrzyński, Croat. Chem. Acta 2017, 90, 75-85. https://doi.org/10.5562/cca3099

[8] N. Trinajstić, Chemical Graph Theory, 2nd ed.; CRC Press: Boca Raton, Florida, USA, 1992.

[9] R. B. Mallion, Croat. Chem. Acta 2008, 81, 227-246. https://hrcak.srce.hr/28146

[10] A. T. Balaban, T. K. Dickens, I. Gutman, R. B. Mallion, Croat. Chem. Acta 2010, 83, 209-215. https://hrcak.srce.hr/56024

[11] C. A. Coulson, B. O'Leary, R. B. Mallion, Hückel Theory for Organic Chemists, Academic Press, London, 1978.

[12] K. Yates, Hückel Molecular Orbital Theory, Academic Press, New York, 1978. https://doi.org/10.1016/B978-0-12-768850-3.50005-5

[13] F. London, J. Phys. Radium (7e Série) 1937, 8, 397-409. https://doi.org/10.1051/jphysrad:01937008010039700

[14] J. A. Pople, Mol. Phys. 1958, 1, 175-180. https://doi.org/10.1080/00268975800100211

[15] R. McWeeny, Mol. Phys. 1958, 1, 311-321. https://doi.org/10.1080/00268975800100381

[16] T. K. Dickens, R. B. Mallion, Croat. Chem. Acta 2013 $86,387-406$, especially p. 390. https://doi.org/10.5562/cca2291

[17] T. K. Dickens, R. B. Mallion, MATCH Commun. Math. Comput. Chem. 2016, 76, 297-356.
[18] J. A. N. F. Gomes, R. B. Mallion, 'The concept of ring currents', in: D. H. Rouvray (Ed.), Concepts in Chemistry, Research Studies Press Limited, Taunton, Somerset, England, United Kingdom, 1997, and John Wiley \& Sons, Inc., New York, 1997, Chapter 7, pp. 205-253.

[19] J. A. N. F. Gomes, R. B. Mallion, Chem. Rev. 2001, 101, 1349-1384 (especially pp. 1358-1366). https://doi.org/10.1021/cr990323h

[20] F. A. Cotton, Chemical Applications of Group Theory, John Wiley \& Sons, New York and London, 1963, pp. 123-125.

[21] R. B. Mallion, 'The topological nature of the Aufbau process and of quantities calculated via simple molecular-orbital theory', in: A. Graovac, I. Gutman, D. Vukičević (Eds.), Mathematical Methods and Modelling for Students of Chemistry and Biology, Hum naklada d.o.o., Zagreb, 2009, pp. 55-86, especially $\S \S 6 \& 7$, pp. 72-81.

[22] R. B. Mallion, D. H. Rouvray, Mol. Phys. 1978, 36, 125-128. https://doi.org/10.1080/00268977800101451

[23] R. B. Mallion, D. H. Rouvray, Studia Scientiarum Mathematicarum Hungarica 1978, 13, 229-243.

[24] A. Ceulemans, E. Lijnen, P. W. Fowler, R. B. Mallion, T. Pisanski, Proc. Royal Soc. (London) Ser. A 2012, 468, 971-989. https://doi.org/10.1098/rspa.2011.0508

[25] T. K. Dickens, R. B. Mallion, RSC Advances 2013, 3, 15585-15588.

https://doi.org/10.1039/c3ra42913

[26] T. K. Dickens, R. B. Mallion, Chem. Commun. 2015, 51, 1819-1822. https://doi.org/10.1039/C4CC07322C

[27] I. Agranat, B. A. Hess, L. Schaad, Pure Appl. Chem. 1980, 52, 1399-1407. https://doi.org/10.1351/pac198052061399

[28] G. Monaco, R. Zanasi, J. Phys. Chem. A 2012, 116, 9020-9026. https://doi.org/10.1021/jp302635j

[29] G. Monaco, R. Zanasi, J. Phys. Org. Chem. 2013, 26, 730-736. https://doi.org/10.1002/poc.3117

[30] T. K. Dickens, R. B. Mallion, J. Phys. Chem. A 2014 118, 933-939. https://doi.org/10.1021/jp411524k

[31] T. K. Dickens, R. B. Mallion, J. Phys. Chem. A 2018, 122, 7666-7678. https://doi.org/10.1021/acs.jpca.8b06862

[32] G. B. Baryshikov, R. R. Valiev, N. N. Karaush, D. Sundholm, B. F. Mineav, Phys. Chem. Chem. Phys. 2016, 18, 8980-8992. https://doi.org/10.1039/C6CP00365F

[33] J. Aihara, J. Am. Chem. Soc. 1979, 101, 5913-5917. https://doi.org/10.1021/ja00514a009 
[34] J. Aihara, T. Horikawa, Bull. Chem. Soc. Jpn 1983, 56, 1853-1854.

https://doi.org/10.1246/bcsj.56.1853

[35] J. Aihara, J. Am. Chem. Soc. 1985, 107, 298-302. https://doi.org/10.1021/ja00288a002

[36] N. Mizoguchi, Bull. Chem. Soc. Jpn 1987, 60, 20052010. https://doi.org/10.1246/bcsj.60.2005

[37] B. O'Leary, R. B. Mallion, J. Math. Chem. 1989, 3, 323-342. https://doi.org/10.1007/BF01169015

[38] J. Aihara, Bull. Chem. Soc. Jpn, 2018, 91, 274-303. https://doi.org/10.1246/bcsj.20170318

[39] W. Kutzelnigg, J. Computat. Chem. 2007, 28, 25-34. https://doi.org/10.1002/jcc.20470

[40] R. B. Mallion, Proc. Royal Soc. (London) Ser. A 1974/1975, 341, 429-449, especially the Appendix (pp. 448-449).

[41] D. J. Klein, C. J. Nelin, S. Alexander, F. A. Matsen, J. Chem. Phys. 1982, 77, 3101-3108. https://doi.org/10.1063/1.444233

[42] D. J. Klein, Pure Appl. Chem. 2008, 80, 1399-1414. https://doi.org/10.1351/pac200880071399

[43] D. J. Klein, Y. Yang, D. Ye, Proc. Royal Soc. (London) Ser. A 2015, 471, 20150183. https://doi.org/10.1098/rspa.2015.0183

[44] J. A. Pople, K. G. Untch, J. Am. Chem. Soc. 1966, 88, 4811-4815. https://doi.org/10.1021/ja00973a009
[45] J. Aihara, Phys. Chem. Chem. Phys. 2016, 18, 1184711857, especially p. 11853. https://doi.org/10.1039/C5CP06471F

[46] A. T. Balaban, D. E. Bean, P. W. Fowler, Acta Chim. Slov. 2010, 57, 507-512. http://acta-arhiv.chem-soc.si/57/57-3-507.pdf

[47] T. K. Dickens, R. B. Mallion, J. Phys. Chem. A 2011, 115, 13877-13884. https://doi.org/10.1021/jp205964t

[48] R. B. Mallion, Mol. Phys. 1973, 25, 1415-1432. https://doi.org/10.1080/00268977300101211

[49] C. W. Haigh, R. B. Mallion, Croat. Chem. Acta 1989, 62, 1-26. https://hrcak.srce.hr/175374

[50] T. K. Dickens, R. B. Mallion, J. A. N. F. Gomes, J. Chem. Theory Comput. 2011, 7, 3661-3674. https://doi.org/10.1021/ct2002539

[51] S. T. Epstein, J. Chem. Phys. 1973, 58, 1592-1595. https://doi.org/10.1063/1.1679398

[52] S. T. Epstein, The Variation Method in Quantum Mechanics, Academic Press, New York, 1974.

[53] M. Kaipio, M. Patzschke, H. Fliegl, F. Pichierri, D. Sundholm, J. Phys. Chem. A 2012, 116, 1025710268. https://doi.org/10.1021/jp308121b

[54] S. Taubert, D. Sundholm, J. Jusélius, J. Chem. Phys. 2011, 134, 054123.

https://doi.org/10.1063/1.3549567 